\title{
AVALIAÇÃO DE UM CHATBOT NO CONTEXTO EDUCACIONAL: UM RELATO DE EXPERIÊNCIA COM METIS
}

Ivana Lima Lucchesi - PPGIE/UFRGS, ivanaufrgs@ gmail.com Anita Raquel da Silva - UFRGS, IFRS, anitarcsg@gmail.com

Cristiane Abreu, cristianeabreu88@ gmail.com Liane Margarida Rockenbach Tarouco_- PPGIE/UFRGS, liane@ penta.ufrgs.br

Resumo. Este artigo relata o experimento de uso do chatbot Metis como apoio às atividades de educação a distância. Apresenta-se relatos sobre o desenvolvimento de chatbot educacional Metis (Mediadora de Educação em Tecnologia Informática e Socializadora) e sobre a estratégia pedagógica de alimentação da base de conhecimento do chatbot, com a participação dos alunos. Descreve-se a avaliação do chatbot sob um viés pedagógico que envolveu a análise das interações e a construção de cinco categorias, construídas a partir de questionário on line. Os resultados mostraram que mesmo em fase inicial de implementação, a agente Metis alcançou resultados satisfatórios quanto à eficiência de diálogo e influência no engajamento dos alunos, na turma investigada.

Palavras-chave: chatbots; agentes conversacionais; base de conhecimento.

\section{EVALUATION OF A CHATBOT IN THE EDUCATIONAL CONTEXT: AN EXPERIENCE WITH METIS}

Abstract: This article reports on the experiment of using the Metis chatbot as a support for distance education activities. There are reports on the development of educational chatbot Metis (Education and Information Technology and Socialization Technology) and on the pedagogical strategy of feeding the chatbot knowledge base, with the participation of the students. We describe the chatbot evaluation under a pedagogical bias that involved the analysis of the interactions and the construction of five categories, constructed from an online questionnaire. The results showed that even in the initial phase of implementation, the agent Metis achieved satisfactory results regarding the efficiency of dialogue and influence in the students' engagement in the group investigated.

Keywords: chatbots; conversational agents; knowledge base.

\section{Introdução}

Na passagem de gerações, o fomento à interação do homem com a máquina e o produto desta relação têm tornado disponíveis recursos tecnológicos inovadores, que fornecem um suporte adicional às práticas educativas formais - os chatbots.

Chatbots são agentes de conversação, ou melhor, aplicativos inteligentes que simulam a conversação como se fossem seres humanos. Resultante dos avanços das pesquisas da Inteligência Artificial (I.A.), muitos projetos de "bots", cada vez mais robustos, estão sendo projetados e desenvolvidos a desempenhar um conjunto de funções humanas, como as de comunicação, raciocínio, aprendizagem, reconhecimento de padrões e comportamentos e a inferência em situações do cotidiano, tornando-se um facilitador de conteúdos de interação (GARTNER'S, 2017). 
No campo educacional, esta tendência expande-se, revelando distintos interesses nos efeitos dos chatbots no processo pedagógico. Experiências iniciais como as descritas em Leonhardt et al., (2003) relatam o uso do ambiente ALICE na criação do chatbot Elektra, proposta para atuar como uma professora para apoio ao ensino da Física, que responde perguntas e direciona o usuário a websites validados no banco de dados da agente. Outro exemplo de uso de chatbot no país está voltado para a interação do usuário por meio de bate-papo em ambientes de aprendizagem, resultou na criação do chatbot Lassalinho (GOMES et al. (2005). Paschoal et al, (2016) projetaram Ubibot para interagir no ambiente Moodle. Tais autores vêm a interatividade proporcionada pelos chatbot educacionais como fonte de motivação e oportunidade para ensejar interação capaz de promover aprendizagem.

Avanços tecnológicos, elicitados pelo relatório Hype Cycle for Emerging Technologies 2017 (GARTNER'S, 2016), ciclo anual de perspectivas sobre as tecnologias, apontam o crescimento de interfaces conversacionais com o usuário no período de cinco a dez anos, estando esta tecnologia situada, na avaliação de 2016, ainda no patamar de disparados de inovações. A Figura 1 resume as expectativas em termos de tecnologias em desenvolvimento e os prazos de amadurecimento esperados.

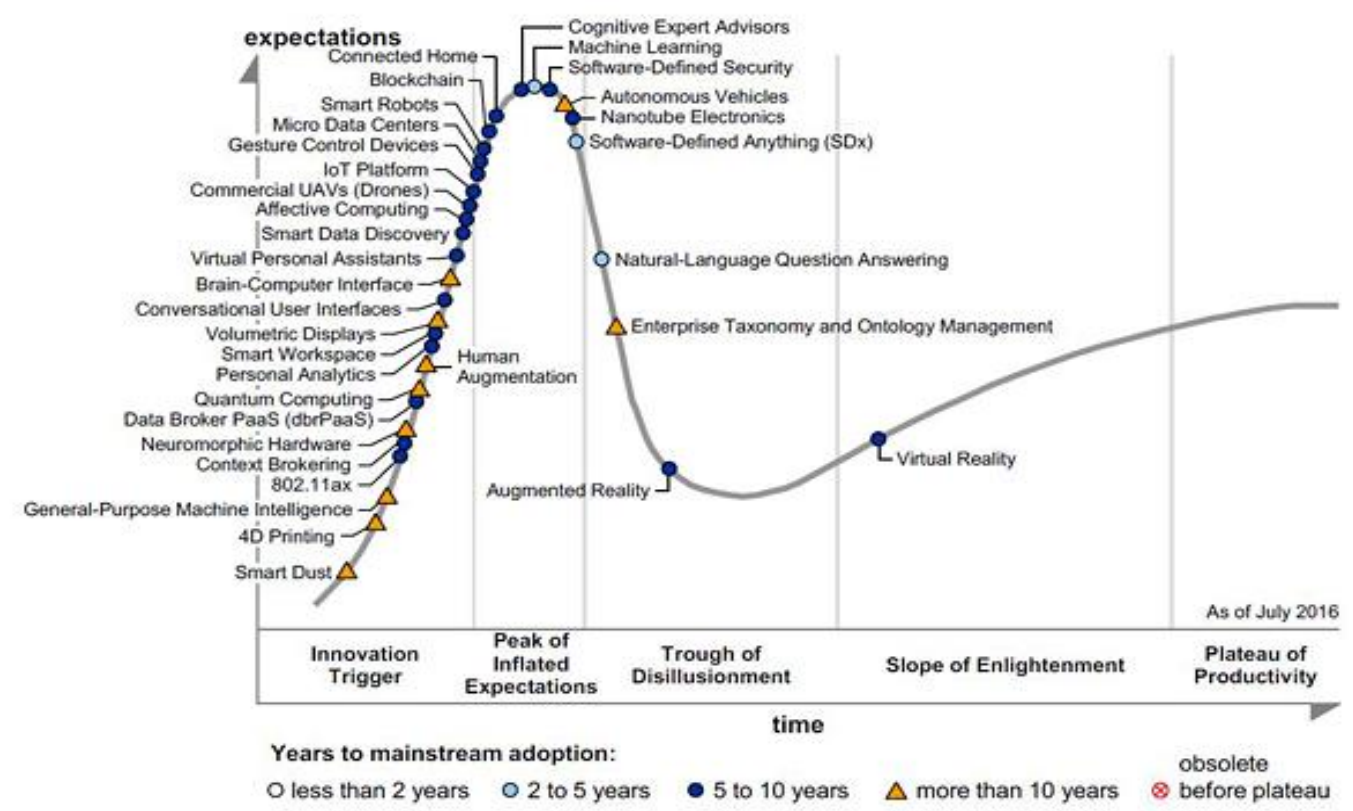

Source: Gartner (huly 2016)

Figura 1: Hype Cycle para tecnologias emergentes em 2016

Fonte: Gartner (2016) ${ }^{1}$.

No entanto, os sistemas de pergunta e resposta em linguagem natural, que têm uma expectativa de adoção no prazo de dois a cinco anos, foram situados no patamar do vale de desilusão indicando ter havido muita expectativa naquela particular tecnologia. Investimentos promoveram o retorno esperado e muitos pioneiros retiraram-se no cenário, estando à tecnologia necessitando melhorias que permitam a obtenção de melhores resultados com menor custo. A tecnologia de aprendizagem de máquina está posicionada no topo das expectativas no ciclo de 2016, indicando ser uma tecnologia emergente da qual muito se comenta, casos de sucesso são relatados, mas também há casos de insucesso. Esta tecnologia permitirá aprimorar de forma semi-automatizada a

1Disponível em: < https://www.gartner.com/newsroom/id/3412017> . Acesso em: 28 out. 2017. 
capacidade de compreensão necessária às interfaces conversacionais baseados em linguagem natural típico dos chatbots e ocasionar a intensificação e disseminação de seu uso?

Segundo pesquisas do Chatbots Journal ${ }^{2}$, até o ano de 2020, a tendência de tecnologia estratégica com foco em sistemas conversacionais chegará a mais de $85 \%$ dos centros de atendimento ao cliente, com "bots" capazes de atendimento personalizado e reconhecimento de voz, face e comportamento de compra do cliente.

Neste sentido, este artigo relata o experimento de uso do chatbot Metis como apoio às atividades de educação a distância. Serão apresentados relatos sobre $\mathrm{o}$ desenvolvimento do chatbot educacional Metis - Mediadora de Educação em Tecnologia Informática e Socializadora - e sobre a estratégia pedagógica de alimentação da base de conhecimento do chatbot, com participação dos alunos. Descreve-se a avaliação do chatbot sob um viés pedagógico que envolveu a análise das interações e a construção de categorias, construídos a partir de questionário on line. Os principais resultados, bem como as dificuldades encontradas serão analisados e discutidos.

\section{CHATBOTS: visão geral}

$\mathrm{O}$ Teste de Turin, retratado na obra "Computing, Machinery and Intelligence" (TURING, 1950), teve como conceito fundamental os princípios da Inteligência Artificial (HODGES, 2012). O teste original de Turing, lançado pelo matemático Alan Turing em 1950, avaliava a conversação entre dois participantes e uma máquina, os quais teriam de manter um diálogo, de maneira que a máquina se mostrasse indistinguível na avaliação à resposta de um humano. Posteriormente, outros programas, como Eliza (WEIZENBAUM, 1966), Shrdlu (WINOGRAD, 1970), Parry (COLBY, 1972) e Alice (WALLACE, 2003) tornaram-se pioneiros da história da informática.

O chatbot Eliza (WEIZENBAUM, 1966), um agente conversacional clássico, criado pelo cientista da computação Joseph Weizenbaum em 1966, representa um dos mais antigos e conhecidos chatbot da história da informática. O programa ELIZA fornecia aos usuários uma ilusão de compreensão por parte do programa, mas assim como muitos dos atuais chatbots, Eliza não tinha estrutura construída para contextualizar.

O programa SHRDLU (WINOGRAD, 1970) idealizado por Terry Winograd, em 1970 teve como característica principal o modelo de conversação desenvolvido para comandar operações com blocos num "mundo dos blocos", objetos e coleções. A combinação dos blocos básicos de construção da linguagem permitia uma linguagem simples e a dedução de como os blocos poderiam ser empilhados (BLOM \& THORSEN, 2013).

PARRY (COLBY, 1972) foi o chatbot inovador dentro da concepção de Inteligência Artificial. Ele foi posto a interagir com o programa ELIZA em diversas oportunidades sendo uma das mais notáveis a interação na International Conference on Computer Communications (ICCC 1972) quando foi estabelecida uma conexão através da Arpanet para a interação. Foi o primeiro programa a passar no Teste de Turing quando interrogadores humanos não conseguiram distinguir Parry de um indivíduo paranoico real.

2 Disponível em: < https://chatbotsjournal.com/ > . Acesso em: 25 ago. 2017. 
A.L.I.C.E. (Artificial Linguistic Internet Chat Entity) foi considerado um dos programas mais populares na atualidade. Este software ganhou o prêmio Loebner, prêmio de reconhecimento ao chatbot mais humano no campo da Inteligência Artificial, caracterizado por manter uma conversação com o usuário de maneira a enganá-lo ou convencê-lo de estar falando com um humano. O programa A.L.I.C.E foi projetado por Richard Wallace (WALLACE, 2003) e desenvolvido pela comunidade de software livre entre os anos de 1995 e 2000.

\subsection{Uso dos chatbots na educação}

O objetivo principal dos agentes conversacionais é instigar um diálogo, de maneira natural, simulando uma comunicação entre pessoas. A naturalidade do processamento da linguagem é um dos principais desafios para os desenvolvedores de chatbots, visto que apresenta caráter motivacional e induz o interlocutor a continuar a interação.

Variáveis motivacionais na área da tecnologia educacional foram revisadas por Heidig \& Clarebout (2011). Os autores afirmam que o uso de agentes no contexto educacional torna-se meio facilitador da aprendizagem e apoio às tarefas de autoregulação, influenciados por variáveis motivacionais como, objetivos de realização e valorização da aprendizagem, no entanto, os benefícios alcançados subordinam-se as condições de uso: (i) características do ambiente de aprendizagem; (ii) conteúdo; (iii) público; (iv) características dos alunos; (v) função e design do agente; visto que, efeitos negativos, como o desprezo ao agente e falta de curiosidade, também podem ser produzidos.

As condições de uso do agente Blaze (AGUIAR, 2011) foram monitoradas e as atividades realizadas possibilitaram os processos de auto-regulação dos alunos. As informações contidas na base de conhecimento do agente educacional Blaze, inseridas por meio da linguagem de marcação AIML (Artificial Intelligence Markup Language), foram construídas pelo conjunto da representação de processos cognitivos de estudantes talentosos, medalhistas da Olimpíada Brasileira de Matemática das Escolas Públicas. Os resultados alcançados na intervenção com o chatbot mostrou o aprimoramento das habilidades cognitivas de resolução de problemas e reações positivas nos estudantes como o interesse (HIDI \& RENNINGER, 2006) e engajamento (BENOTTI et al, 2014).

O programa Doroty (LEONHARDT e TAROUCO, 2005) foi projetado para encorajar as tomadas de decisão dos profissionais de rede que não tiveram treinamento suficiente na área de gerência de redes. Doroty processava os questionamentos dos profissionais e contextualizava as explicações com dados reais extraídos de um ambiente de rede existente.

A inserção de um agente conversacional educacional no ambiente EAD, como a professora Elektra (LEONHARDT et al, 2003) pode ser uma alternativa para minimizar a sensação de falta de contato e permitir uma abordagem mais pessoal para o aluno. Elektra é um chatbot de bate-papo, com base na arquitetura de A.L.IC.E (WALLACE, 1995) projetada, inicialmente, para interagir com alunos do ensino secundário para fins de apoiar a aprendizagem da Física. Posteriormente, suas funcionalidades foram expandidas no intuito de prover atendimento contínuo aos alunos remotos, por meio de outras funcionalidades, como hiperlinks e múltiplas respostas para cada categoria. A professora virtual Elektra está disponível para conexão no endereço http://penta3.ufrgs.br:2002/ . 


\subsection{Agente Conversacional Metis}

A agente conversacional Metis (Mediadora de Educação em Tecnologia Informática e Socializadora) foi projetada para conversar com os alunos por meio de uma interface que simula uma conversação online (chat) entre pessoas. A implementação desta agente utilizou o sistema A.L.IC.E. no qual organiza a base de conhecimento, notação baseada em XML (eXtensible Markup Language). A base de conhecimento do sistema foi alimentada por especialistas em mídias e informática na educação, possibilitando um conhecimento mínimo dos conteúdos e retorno positivo às dúvidas apresentadas pelos alunos. A partir do momento em que os alunos estabeleceram "conversas" com METIS, iniciou-se a inspeção dos registros das interações $(\log s)$, visando identificar perguntas não respondidas pelo sistema. Tais perguntas foram avaliadas e conforme sua relevância, adicionadas à base de conhecimento do agente, ampliando sua capacidade de respostas.

Durante a alimentação e realimentação da base de conhecimento da agente METIS foram introduzidos novos recursos multimídia: (i) adição de imagens nas respostas; (ii) inclusão de links para textos complementares; (iii) links para outros materiais, como vídeos e objetos de aprendizagem externos; (iv) múltiplas respostas para uma mesma pergunta (selecionadas de forma randômica, para que $\mathrm{o}$ comportamento do agente não fosse demasiado determinístico).

\section{Processo metodológico.}

O presente estudo caracterizou-se como qualitativo. Uma amostragem composta por onze alunos foi investigada no primeiro semestre de 2017. A análise de interação entre Metis e os alunos foram comparados com outros dados coletados em outra turma, composta por dez alunos, que cursaram a mesma disciplina em semestre anterior, período no qual a agente conversacional ainda não estava disponível para interação com os alunos.

A coleta de dados foi realizada por meio de três instrumentos: (i) Análise dos logs de conversa registrados pelo chatterbot: trata-se dos logs de conversa registrados entre Metis e os alunos, com o objetivo de avaliar a eficiência dos diálogos. Para tanto, 11 conversas foram analisadas, nas quais cada uma foi enquadrada em um nível de qualidade, concernente à capacidade de responder ou não o usuário satisfatoriamente.

(ii) Análise de acesso aos materiais de apoio da disciplina: Estes dados foram coletados a partir do ambiente virtual de aprendizagem - Moodle - no qual é desenvolvida a disciplina, para análise comparativa entre as duas turmas. Para levantamento e comparação dos acessos dos alunos aos materiais de apoio da disciplina, foi empregado um plugin disponibilizado no Moodle. Tal recurso permite a extração do número de visualizações nos recursos disponíveis no ambiente, como tutoriais, apresentações, links externos. (iii) Questionário de avaliação do agente: Os questionários, por meio de perguntas fechadas, são considerados meios eficazes para apurar informações precisas dos investigados. Neste sentido, foi proposto aos alunos da turma investigada um questionário online, composto por 26 questões objetivas, organizadas em 5 categorias: aprendizagem, confiabilidade, relações, engajamento e visão geral.

\section{Análise e resultados encontrados}

\subsection{Análise dos Logs de Conversa Registrados pelo Chatterbot}


As conversas registradas pelo chatterbot ocorreram no período de oito semanas. Neste período, Metis apresentava aproximadamente 2.800 categorias, atualmente conta com 4.300 categorias em sua base de conhecimento. Foram analisadas 11 conversas, originárias de 11 endereços IP distintos, confirmando usuários diferentes. Os logs registrados resultaram em um total de 117 linhas, com média de 10 linhas por conversa. Os $\log s$ foram classificados por endereço IP e, após, cada diálogo (correspondente a um usuário diferente) foi avaliado quanto a sua eficiência apresentando os seguintes níveis de qualidade: (i) Nível 0 - A agente forneceu majoritariamente respostas que não atenderam à consulta do usuário; (ii) Nível 1 - A agente forneceu respostas que atenderam parcialmente à consulta do usuário (solicitou explicações ou reformulações das perguntas); (iii) Nível 2 - Todas as respostas da agente atenderam à consulta do usuário. Quanto mais baixo o nível de qualidade, menos o chatterbot entendeu e respondeu satisfatoriamente o aluno durante o diálogo. A qualidade aumenta na medida em que o aluno tenha recebido majoritariamente ou exclusivamente respostas que atenderam suas questões.

Gráfico 1 - Eficiência de diálogo a partir dos registros de logs

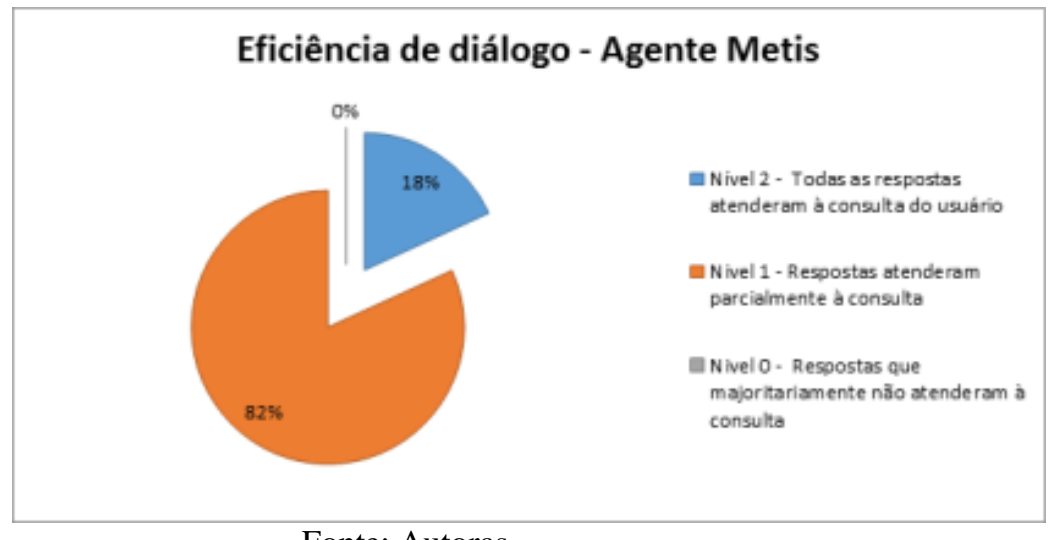

Fonte: Autoras

A partir da verificação dos $\log s$, constatou-se que a maior parte dos diálogos se enquadrou no nível um de qualidade, no qual a agente conversacional forneceu respostas que atenderam parcialmente à consulta dos alunos. Tal nível representou $82 \%$ do total das 11 conversas, como pode ser observado no gráfico um. Por exemplo:

Aluno: Oi Metis!

Metis: oi, como vai?

Aluno: tudo certo. O infográfico pode gerar comparações entre dados?

Metis: fale-me mais sobre isto.

Aluno: gostaria de saber se clicando nas informações do infográfico posso gerar informações novas.

Metis: o que você quer dizer com isso?

Aluno: para que serve um infográfico?

Metis: São representações que utilizam a combinação de fotografia ou desenho e texto. Um exemplo de infográfico do tipo mais simples poderia ser uma linha de tempo onde, ao selecionar determinados períodos, apareceriam imagem e textos explicativos.

No diálogo acima foi possível observar que o chatterbot necessitou de explicações para entender e responder a pergunta sobre infográficos. Em análise de cada 
log enquadrado no nível um, verificou-se os motivos para que Metis não pudesse responder determinadas questões. Após esta análise foram feitos ajustes e inclusões de categorias na base de conhecimento da agente Metis. Além disso, embora ainda em fase inicial no período de coleta de dados, nenhum diálogo foi enquadrado no nível 0 de qualidade, ou seja, nenhum aluno recebeu respostas que majoritariamente não atenderam a sua consulta. De encontro, o nível dois de qualidade, no qual todas as respostas recebidas durante o diálogo foram satisfatórias, expressou-se em $18 \%$ do total das 11 conversas, conforme apontado no gráfico um.

\subsection{Análises de Acesso aos Materiais de Apoio da Disciplina}

Para obter a relação detalhada sobre o acesso dos alunos aos materiais de apoio da disciplina, foi utilizado um plugin do Moodle, denominado Analytics Graphs. Tal recurso ofereceu o número de visualizações efetuadas em cada recurso disponibilizado na disciplina, que incluiu apresentações, vídeos, textos de apoio, tutoriais, links externos, etc. A partir disto, foi possível extrair o número total de visualizações em cada tópico (semanal) estudado, comparando-o nas duas turmas. Dos 14 tópicos semanais analisados e comparados entre as duas turmas, em dois ocorreram acessos em maior quantidade na turma do segundo semestre de 2016, correspondente aos alunos sem acesso à Metis, conforme Gráfico 2. Desta forma, apresentando 12 tópicos com maior número de acessos, a turma que cursou a disciplina no segundo semestre de 2017 (com acesso à Metis) teve um aumento de aproximadamente $22 \%$ no total de acessos aos materiais de apoio. Neste contexto, ponderando que a agente Metis fornece recursos da disciplina, ao responder dúvidas dos alunos, considerou-se que a mesma possa ter contribuído nestes resultados.

\subsection{Questionário de Avaliação do Agente}

O instrumento (iii) foi composto por vinte e seis questões objetivas, organizadas em cinco categorias: aprendizagem (treze questões); confiabilidade (cinco questões) relações (três questões), engajamento (três questões) e visão geral (duas questões). Todas as questões apresentaram opção de resposta tipo escala Likert de cinco pontos, indicando como extremos: discordo totalmente (1) e concordo totalmente (5). A utilização do questionário visou complementar os dados coletados, quanto à usabilidade do agente e seu efeito no engajamento dos estudantes. No intuito de averiguar a confiabilidade do questionário, utilizou-se o coeficiente Alfa de Cronbach para medir a correlação entre as respostas dadas pelos participantes. Como resultado de confiabilidade, obteve-se $\boldsymbol{\alpha}=\mathbf{0}, \mathbf{9 5 2 4}$, considerado uma alta confiabilidade. Dentro do escopo da pesquisa, oito (8) dos onze (11) alunos da disciplina responderam o questionário online, no período de quatro semanas. A seguir serão apresentadas as análises dos resultados obtidos em cada uma das categorias do questionário.

\subsubsection{Categoria Aprendizagem}

A categoria Aprendizagem teve por objetivo avaliar a contribuição do agente conversacional na aprendizagem. Com relação aos participantes que responderam as 13 questões relacionadas à categoria Aprendizagem, um percentual de 37,36\% discordaram parcialmente e $30,77 \%$ ficaram neutros sobre terem construído algum aprendizado com as respostas obtidas às perguntas realizadas à Metis. $\mathrm{O}$ fato de interagir ou testar $\mathrm{O}$ chatterbot pode ser mais interessante para o aluno do que estudar ou tirar dúvidas sobre 
determinado assunto. Ainda, embora não tenha sido um resultado satisfatório, verificamos nas subseções anteriores, um aumento de $22,47 \%$ no acesso a estes materiais e aumento na média final alcançada pelos alunos.

\subsubsection{Categoria Confiabilidade}

A confiabilidade de um material ou informação advém de fontes seguras e fidedignas das quais eles são selecionados. As cinco questões relacionadas à confiabilidade e materiais apresentados pela agente conversacional tiveram como objetivo verificar o percentual de confiabilidade dos participantes com relação ao que lhes era indicado por Metis. Os resultados mostraram um percentual de 37,14\% que concordaram parcialmente e $25,71 \%$ que concordaram totalmente que as respostas e materiais indicados pelo chatterbot são confiáveis como respostas e matéria de consulta.

\subsubsection{Categoria Relações}

O fator relações procura identificar se o chatterbot consegue desenvolver uma conversa muito próxima a linguagem natural com os participantes, demonstrando-se amistoso aos questionamentos realizados pelos mesmos. Tal categoria apresentou um percentual de $38,10 \%$ dos participantes que concordaram parcialmente e $14,29 \%$ que concordaram totalmente quanto ao diálogo amigável, durante as trocas realizadas com a agente Metis.

\subsubsection{Categoria Engajamento}

Embora o chatterbot mostrou-se amistoso durante as trocas e indicações de materiais, Metis precisa envolver o participante de forma que as trocas de informações sejam mais abrangentes e contínuas. Este tipo de engajamento tem relação ao tipo de respostas fornecidas pela agente conversacional, na qual é possível apresentar indagações ou questões reflexivas sobre o tema abordado pelo participante. Um percentual de 33,33\% dos participantes concordaram parcialmente e $23,81 \%$ ficaram neutros em relação a agente Metis ter estimulado a atenção sobre o conteúdo, promovendo assim novos questionamentos.

\subsubsection{Categoria Visão geral}

Com relação as experiências do usuário com o chatterbot e a possibilidade de utilizá-lo futuramente, o percentual apresentado foi o de neutralidade, com 42,86\%, seguido dos que concordam parcialmente, com 21,43\%. A partir dos resultados extraídos do questionário, percebeu-se que os alunos transitaram entre a neutralidade e a concordância parcial, ao avaliar a agente Metis. Embora nenhuma categoria tenha sido avaliada exclusivamente com pontuação 1 (discordo totalmente), verifica-se a necessidade de melhorias no funcionamento da agente conversacional.

\section{Conclusões}

Este artigo apresentou um experimento de uso do chatbot Metis (Mediadora de Educação em Tecnologia Informática e Socializadora) como apoio às atividades de educação a distância. Foram apresentados relatos sobre o desenvolvimento de chatbot educacional Metis e sobre a estratégia pedagógica de alimentação da base de conhecimento do chatbot, com a participação dos alunos. Os resultados mostraram que 
houve um aumento, ainda que moderado, da média geral alcançada pelas duas turmas analisadas. Enquanto a turma de 2016 concluiu o semestre com média 8,2, a turma de 2017 obteve a média 8,6. Infere-se que este aumento esteja relacionado à maior inspeção dos materiais de apoio da disciplina, pois quanto mais aprofundados os recursos, melhor torna-se o embasamento para realização das atividades propostas. Conclui-se que, mesmo em fase inicial de implementação, a agente Metis alcançou resultados satisfatórios quanto à eficiência de diálogo e influência no engajamento dos alunos, na turma investigada, no entanto, sugerem-se melhorias no funcionamento da agente conversacional em futuros estudos. Contudo, a natureza dinâmica dos agentes conversacionais torna-se uma ferramenta próspera no contexto educacional em virtude do sistema rápido de diálogo e pelas possibilidades de interação que podem ser adaptadas, beneficiando, principalmente aprendentes da educação à distância.

\section{Referências}

AGUIAR, Eliane Vigneron Barreto. Aprimoramento das habilidades cognitivas de resolução de problemas com o apoio de um agente conversacional. 2011.

BENOTTI, Luciana; MARTÍNEZ, María Cecilia; SCHAPACHNIK, Fernando. Engaging high school students using chatbots. In: Proceedings of the 2014 conference on Innovation \& technology in computer science education. ACM, 2014. p. 63-68.

COLBY, Kenneth Mark et al. Turing-like indistinguishability tests for the validation of a computer simulation of paranoid processes. Artificial Intelligence, v. 3, p. 199-221, 1972.

Gartner,Inc. (NYSE: IT) http://www.gartner.com/newsroom/id/3412017 .

GOMES, Romualdo Santos; BARBOSA, Débora Nice Ferrari; GEYER, Cláudio Fernando Resin. Lassalinho: um agente pedagógico animado em um ambiente multiagente para educação a distância. RENOTE: revista novas tecnologias na educação [recurso eletrônico]. Porto Alegre, RS, 2005.

HEIDIG, Steffi; CLAREBOUT, Geraldine. Do pedagogical agents make a difference to student motivation and learning?. Educational Research Review, v. 6, n. 1, p. 27-54, 2011.

HIDI, Suzanne; RENNINGER, K. Ann. The four-phase model of interest development. Educational psychologist, v. 41, n. 2, p. 111-127, 2006.

LEONHARDT, Michelle Denise; NEISSE, Ricardo; TAROUCO, Liane Margarida Rockenbach. MEARA: um Chatterbot temático para uso em ambiente educacional. In: Brazilian Symposium on Computers in Education (Simpósio Brasileiro de Informática na Educação-SBIE). 2003. p. 81-88.

LEONHARDT, Michelle Denise; TAROUCO, Liane Margarida Rockenbach. Aplicando linguagem natural ao gerenciamento de redes de computadores através do chatterbot Doroty. In: Congresso da Sociedade Brasileira de Computacao (25.: 2005: São Leopoldo, RS). A universalidade da Computação: um agente de inovação e desenvolvimento: anais [recurso eletrônico]. [Porto Alegre]: SBC, 2005. 2005. 
PASCHOAL, Leo Natan; CHICON, Patricia M. Mozzaquatro; FALKEMBACH, Gilse Antoninha Morgental. Ubibot: um Agente Conversacional Ciente do Contexto de Aprendizagem do Usuário. RENOTE, v. 14, n. 1, 2016.

TURING, A. M. Mind. Mind, v. 59, n. 236, p. 433-460, 1950.

WALLACE, Richard. The elements of AIML style. Alice AI Foundation, 2003.

WEIZENBAUM, Joseph. ELIZA - a computer program for the study of natural language communication between man and machine. Communications of the ACM, v. 9, n. 1, p. 36-45, 1966.

WINOGRAD, Terry. Procedures as a representation for data in a computer program for understanding natural language. MASSACHUSETTS INST OF TECH CAMBRIDGE PROJECT MAC, 1971. 